\title{
Two Cases of Vulvovaginitis Caused by Shigella flexneri and Shigella sonnei: a Case Report
}

\author{
Gülçin Bayramoğlu¹, Faruk Aydın, Gülay Karagüzel², Mustafa İmamoğlu³, Ayşenur Ökten² \\ ${ }^{1}$ Department of Medical Microbiology, Faculty of Medicine, Karadeniz Technical University, Trabzon, Turkey \\ ${ }^{2}$ Department of Pediatrics, Faculty of Medicine, Karadeniz Technical University, Trabzon, Turkey \\ ${ }^{3}$ Department of Pediatric Surgery, Faculty of Medicine, Karadeniz Technical University, Trabzon, Turkey
}

\begin{abstract}
Vulvovaginitis caused by Shigella species (Shigella spp.) has rarely been reported. This paper describes two cases of prepubertal vulvovaginitis, presenting with a bloody and purulent vaginal discharge, separately caused by ampicillin-resistant Shigella flexneri and trimethoprim-sulfomethoxazoleresistant Shigella sonnei. Our conclusions are that Shigella spp. is the potential cause of vulvovaginitis in prepubertal girls in developing countries where these pathogens are endemic, and identification of the bacteria and making antibiotic susceptibility testing in these cases should not be overlooked.
\end{abstract}

Key Words: Shigella flexneri, Shigella sonnei, vulvovaginitis, prepubertal girls

Received: 09.10.2010

Accepted: 07.12.2010

\section{Introduction}

Shigellosis or bacillary dysentery is a global human health problem with an estimated annual incidence of 164.7 million cases, of which 163.2 million occur in developing countries, and causes 1.1 million deaths (1). Shigella species are not part of the normal human flora, and are well-recognized agents of acute bacillary dysentery (2).Vulvovaginitis caused by Shigella spp. have been rarely reported (3-9). This may be partly due to the fact that diagnosis of Shigella vulvovaginitis may be difficult. Most of these cases are reported in children (3-9).

We describe here two cases of vulvovaginitis caused by Shigella flexneri and Shigella sonnei, and discuss some problems of the diagnosis and treatment of vulvovaginitis caused by Shigella spp.

\section{Case Reports}

\section{Case 1}

A nine-year-old girl was referred to our department for further evaluation of dyslipidemia and purulent vaginal discharge of unknown etiology. She had a history of a bloody-purulent vaginal discharge, and vulvovaginal itching for two months. There were no findings of any sexual abuse, history of serious illness and hospitalization, or the presence of any foreign body.

Vulvovaginal examination showed mucosal erythema with a yellowish purulent vaginal discharge mixed with blood. Gram stain smear of the vaginal discharge showed numerous polymorphonuclear leucocytes, gram-negative bacilli and predominant growth of Escherichia coli. No intracellular gram-negative diplococci were seen at microscopic examination. The urine culture was negative. Based on the diagnosis of nonspecific vaginitis, the patient was advised on local hygienic measures. Since $E$. coli growth might have been due to contamination from the perianal skin, vaginal culture was repeated five days later and $S$. flexneri was identified. The third vaginal culture was again positive for $S$. flexneri. The stool cultures of the patient and other family members were negative for S. flexneri. The $S$. flexneri isolate was susceptible to ceftriaxone, ceftazidime, cefepime, ciprofloxacin, levofloxacin, chloramphenicol, trimethoprim-sulfamethoxazole, imipenem, meropenem and tigecycline and resistant to ampicillin and amoxicillin-clavulanic acid. The patient was treated with oral trimethoprim-sulfamethoxazole for 10 days, and followup vaginal cultures were found negative.

\section{Case 2}

An eight-year-old girl presented at our department with a one-year history of intermittent foul smelling, yellowish purulent vaginal discharge and severe vulvovaginal itching. The discharge had turned bloody during the last two months. Although treated elsewhere with unknown oral medication for one year, her symptoms had not improved. No other health problem, sexual abuse or trauma was reported.

The physical examination at admission showed a reddish vaginal mucosa and bloody-vaginal discharge. No vaginal foreign body was detected. S. sonnei resistant in vitro to trimethoprim-sulfamethoxazole but susceptible to ampicillin, amoxicillin-clavulanic acid, ceftriaxone, ceftazidime, cefepime, ciprofloxacin, levofloxacin, chloramphenicol, imipe- 
nem, meropenem and tigecycline grew in the vaginal culture. The Gram-stained smear of the vaginal discharge showed numerous polymorphonuclear leucocytes but no gram-negative diplococci. The stool cultures of the patient and family members were negative for Shigella spp. and any other enteric pathogens. She was treated orally with amoxicillin-clavulanic acid for 10 days. The vaginal discharge was not resolved after 10 days of the treatment. On the third day after completion of antibiotic therapy, S. sonnei at 100 cfu per milliliter with the same susceptibility pattern was isolated from urine, but the repeated cultures from the vaginal discharge were negative. No further culture was performed since the patient made no further visits to our clinic for a month. When she returned after one month, vaginal culture had remained positive, and stool and urine cultures were negative for $S$. sonnei. The patient was prescribed oral cefixime for 10 days, and at follow-up examination, the vaginal culture was positive for $S$. sonnei. The patient was given a 14-day course of ciprofloxacin. The vaginal discharge resolved after the treatment with ciprofloxacin. Follow-up vaginal cultures were negative.

\section{Discussion}

Chronic vaginal discharge is the most frequent finding in Shigella vulvovaginitis and, as also noted with our cases, is resolved in longer than 10 days. The purulent-mucopurulent foul smelling discharge, varying from whitish to yellowish to greenish, does not differ from vulvovaginitis caused by the other microorganisms, however, the bloody discharge could be an indication for Shigella vulvovaginitis $(5,9)$. As shown in Table 1, eight out of 11 cases (73\%), including ours, presented with bloody-vaginal discharge. Here no significant difference between $S$. flexneri and $S$. sonnei infection regarding the frequency of bloody discharge has been detected, although bloody diarrhea due to $S$. flexneri has been reported more often compared to that of S. sonnei (9).

Laboratory diagnosis of Shigella vaginal infections is not easy. One of the possible reasons is that a request for the identification of Shigella is not common since many microbiology laboratories may not identify gram-negative rods recovered from vaginal cultures $(4,7)$. For instance to isolate and differentiate Shigella spp. in samples containing bacterial flo$\mathrm{ra}$, it is advised that clinical sample should be inoculated onto Salmonella Shigella (SS) agar, eosin-methylene blue (EMB) and selenite broth (SB) and incubated for longer times (10). None of these growth media are mentioned among the media suggested for routine culturing of vaginal samples. When it has been done, the suggested media for vaginal specimens of children are $5 \%$ sheep blood agar and chocolate agar for Streptococcus pyogenes, Streptococcus pneumoniae and Haemophilus influenzae, Sabouraud medium for Candida spp. and selective gonococcal medium for Neisseria gonorrhoeae. Because, Shigella spp. and other gram-negative bacilli are not considered as possible vulvovaginitis pathogens in prepubertal girls, (11) and gram-negative rods (such as Proteus species, Pseudomonas species) known to occur in healthy prepubertal girls without causing any illness (12). Additionally, Gram-negative rods of fecal origin can also be found as contaminants. As a result, Shigella spp. is easily overlooked in routine vaginal cultures if no pure or prominent growth was detected. Another reason for missing Shigella in laboratory diagnosis is inaccurate specimen collection and transportation. Shigella spp. are fragile organisms and remain viable for a limited time outside the human body. Therefore, considerable care must be taken in the collection and transportation of the clinical specimens, and informing the laboratory about the possible causative

Table 1. Reported cases (Including the Present Cases) of Shigella vulvovaginitis. At the far left column, the number of the cases; at the top line, the features of the cases have been shown.

\begin{tabular}{|c|c|c|c|c|c|c|c|c|c|}
\hline Case & Year & Ref. & Age & $\begin{array}{l}\text { Shigella } \\
\text { species }\end{array}$ & $\begin{array}{l}\text { Bloody } \\
\text { discharge }\end{array}$ & $\begin{array}{c}\text { Associated } \\
\text { esymptoms }\end{array}$ & $\begin{array}{l}\text { Antibiotic } \\
\text { resistance pattern }\end{array}$ & $\begin{array}{l}\text { Ineffective } \\
\text { treatment }\end{array}$ & $\begin{array}{l}\text { Effective } \\
\text { treatment }\end{array}$ \\
\hline 1 & 1950 & (3) & 7 & S. flexneri & No & No & ND & No & Sulphaguanidine orally \\
\hline 2 & 1975 & (4) & 6 & S. flexneri & Yes & No & ND & No & Ampicillin orally \\
\hline 3 & 1975 & (4) & 4 & S. flexneri & Yes Ur & $\begin{array}{l}\text { Urinary frequency } \\
\text { and dysuria }\end{array}$ & ND & No & Ampicillin orally \\
\hline 4 & 1975 & (4) & 4 & S. flexneri & Yes & Dysuria & ND & No & Ampicillin orally \\
\hline 5 & 1975 & (4) & 6 & S. flexneri & Yes & No & ND & $\begin{array}{l}\text { Nitrofurantoin, } \\
\text { ampicillin orally }\end{array}$ & $\begin{array}{c}\text { Estrogen/ } \\
\text { sulfasoxazole intravaginally }\end{array}$ \\
\hline 6 & 1979 & (5) & 4 & S. sonnei & No & No & Ampicillin & Ampicillin orally & $\begin{array}{c}\text { Estrogen/ } \\
\text { sulfasoxazole intravaginally }\end{array}$ \\
\hline 7 & 2002 & (6) & 7 & S. flexneri & Yes & Dysuria & Ampicillin, SXT & $\begin{array}{l}\text { SXT, AMC, } \\
\text { cefixime orally }\end{array}$ & Ciprofloxacin orally \\
\hline 8 & 2002 & (7) & 5 & S. flexneri & Yes & No & No resistance & No & SXT orally \\
\hline 9 & 2006 & (8) & 5 & S. flexneri & No & Dysuria & Ampicillin, SXT & No & Cefixime orally \\
\hline $10 *$ & 2009 & & 9 & S. flexneri & Yes & No & Ampicillin, AMC & No & SXT orally \\
\hline $11 *$ & 2009 & & 8 & S. sonnei & Yes & No & SXT & $\begin{array}{l}\text { AMC, cefixime } \\
\text { orally }\end{array}$ & Ciprofloxacin orally \\
\hline
\end{tabular}


agents (13). For a fast and accurate diagnosis, the microbiology laboratory should be certainly informed by clinicians if a Shigella infection is considered or if vaginal discharge with blood is present in the patient. The presence of leucocytes in vaginal secretions may be an indicator for clinicians.

Because of the infrequency of the disease, and the limitations in clinical experience, the optimal therapy for Shigella vulvovaginitis remains uncertain $(7,8)$. Systemic antibiotic therapy is generally recommended as being more effective than topical antibiotic therapy $(4,6,8)$. Although the recommended standard course of therapy for shigellosis is oral ampicillin or trimethoprim-sulfamethoxazole therapy, it should be noted that, while initially susceptible, most Shigella isolates are currently resistant to these antibiotics. These are inappropriate for empiric therapy unless local microbiological data suggest susceptibility $(1,6,8)$.

In the treatment of Shigellosis, intracellular concentrations of antibiotics are probably the crucial determinants of success, since Shigella spp. multiply intracellularly. Fluoroquinolones such as ciprofloxacin, and macrolides such as azithromycin, may be particularly useful for the treatment of shigellosis, because, they reach high intracellular levels $(13,14)$. On the other hand, the use of fluoroquinolone in children has been limited because of the concerns about its toxicity. However, there is growing evidence of their safety for the treatment of shigellosis in children $(1,12)$. Azithromycin is approved for use in children $(13,14)$. The significant disadvantage of the azithromycin is the lack of Clinical Laboratory Standards Institute-defined breakpoints for it for the Enterobacteriaceae, including Shigella spp. (15). Susceptibility of the Shigella isolates to azithromycin is not routinely tested in most countries which use Clinical Laboratory Standards Institute documents for antibiotic susceptibility tests, as in Turkey.

In our first case, the S. flexneri isolate was susceptible to a variety of agents including trimethoprim-sulfamethoxazole, and the infection was successfully treated with oral trimethoprim-sulfamethoxazole. In our second case, however, the S. sonnei isolate was susceptible in vitro to amoxicillin/clavulanic acid and cefixime, but the treatments with these antibiotics were not successful. Failure with amoxicillin/clavulanic acid and cefixime was also reported by Baiulescu et al.(6) in a child with Shigella vulvovaginitis, in which the organism was susceptible in vitro to amoxicillin/clavulanic acid and cefixime, but the patient exhibited clinical and microbiologic failure until ciprofloxacin therapy was instituted. Our case and the case reported by Baiulescu et al. (6) differ only in respect to the agents isolated, $S$. sonnei and $S$. flexneri, respectively. The failures in treatment with amoxicillin/clavulanic acid and cefixime suggest a possible difference in the form of infection rather than Shigella species. It is likely that involvement of gynecological tissue complicates the success of the management.
In conclusion, our experience strongly suggests that identification and antibiotic susceptibility testing for Shigella spp., as a potential cause of vulvovaginitis in prepubertal girls presenting with chronic vaginal discharge, especially in developing countries where these pathogens are endemic, should not be overlooked. Additionally, our current understanding suggests that fluoroquinolone antibiotics such as ciprofloxacin might not be the first but nevertheless the best choice against vulvovaginosis for the patients not responding to the regular treatment, and there is not always a good correlation between in vivo clinical response and in vitro antibiotic susceptibility test results.

\section{Conflict of Interest}

No conflict of interest was declared by the authors.

\section{References}

1. Ashkenazi S. Shigella infections in children: new insights. Semin Pediatr Infect Dis 2004;15:246-52. [CrossRef]

2. Farmer JJ. Enterobacteriaceae: introduction and identification. In: Murray PR, Baron EJ, Jorgensen JH, Pfaller MA, Yolken RH, editors. Manual of clinical microbiology. 8th ed. Washington DC: American Society for Microbiology; 2003. p. 636-53.

3. Mcginness WJ, Telling RC. Vulvovaginitis in a child due to Shigella flexneri. Br Med J 1950;23:1424. [CrossRef]

4. Davis TC. Chronic vulvovaginitis in children due to Shigella flexneri. Pediatrics 1975;56:41-4.

5. Rajkumar S, Narayanaswamy G, Laude TA. Shigella vulvovaginitis in childhood: A case report. J Natl Med Assoc 1979;71:1005-6.

6. Baiulescu M, Hannon PR, Marcinak JF, Janda WM, Schreckenberger PC. Chronic vulvovaginitis caused by antibiotic-resistant Shigella flexneri in a prepubertal child. Pediatr Infect Dis J 2002;21:170-2. [CrossRef]

7. Namdari H, Taroli A, Piemontese S. Shigella flexneri as a cause of chronic vaginitis in an Allegedly Sexually Abused Five-Year-Old Girl. Clinical Microbiology Newsletter 2002;24:187-8. [CrossRef]

8. Jasper JM, Ward MA. Shigella vulvovaginitis in a prepubertal child. Pediatr Emerg Care 2006;22:585-6. [CrossRef]

9. Murphy TV, Nelson JD. Shigella vaginitis: report of 38 patients and review of the literature. Pediatrics 1979;63:511-6.

10. Öner $M$, Özer B, Turan P, Şakru N. Dışkı kültürlerinde salmonella cinsi bakterilerin izolasyonunda uzamış inkübasyon süresinin etkisi. Trakya Univ Tıp Fak Derg 2005;22:23-6.

11. Macsween KF, Ridgway GL. The laboratory investigation of vaginal discharge. J Clin Pathol 1998;51:564-7.

12. Stricker T, Navratil F, Sennhauser F H. Vulvovaginitis in prepubertal girls. Arch Dis Child 2003;88:324-6. [CrossRef]

13. Niyogi SK. Shigellosis. J Microbiol 2005;43:133-43. [Review] [CrossRef]

14. Khan WA, Seas C, Dhar U, Salam MA, Bennish ML. Treatment of shigellosis: V. Comparison of azithromycin and ciprofloxacin. A double-blind, randomized, controlled trial. Ann Intern Med 1997;126:697-703.

15. Clinical and Laboratory Standards Institute (CLSI). Performance Standards for Antimicrobial Susceptibility Testing; eighteenth informational supplement. CLSI document M100-S18. Wayne, PA: Clinical and Laboratory Standards Institute; 2008. 